\title{
The Influence of Magnetic Inhomogeneous State on Thermopower and Magnetothermopower in $\mathrm{Sm}_{0.55} \mathrm{Sr}_{0.45} \mathrm{MnO}_{3}$ Manganites
}

\author{
L. Koroleva $^{1, \mathrm{a}}$, A. Morozov ${ }^{1}$, and E. Zhakina ${ }^{1}$ \\ ${ }^{1}$ Physics Department, Moscow State University, Moscow, Russia
}

\begin{abstract}
Thermopower $\alpha$ and magnetothermopower $\Delta \alpha / \alpha$ were studied in the single-crystal $\mathrm{Sm}_{0.55} \mathrm{Sr}_{0.45} \mathrm{MnO}_{3}$ samples, containing clusters of following three types: ferromagnetic clusters with the Curie temperature $T_{C}=$ $134 \mathrm{~K}$, A-type antiferromagnetic clusters with the Neel temperature $T_{N A} \leq T_{C}$ and CE-type antiferromagnetic clusters with the $T_{N C E}=240 \mathrm{~K}$. Temperature dependence of $\alpha$ and $\Delta \alpha / \alpha$ have extrema in the $T_{N C E}$-region: large maximum on $\alpha(T)$ and sharp minimum on $\{\Delta \alpha / \alpha\}(T)$. Negative magnetothermopower in minimum achieves the giant value $50 \%$ in magnetic field $H=13,2 \mathrm{kOe}$. As will be shown below thermopower is essentially caused by the presence of CE-type antiferromagnetic clusters, in which exists charge order (CO), displacing oxygen ions. Modified crystalline lattice inside clusters causes change of thermopower in them. This thermopower influences on the voltage drop on sample at measurement of thermopower and, consequently, on the effective value $\alpha$ of the whole sample. Enclosure of the magnetic field in the $T_{N C E}$, which accelerates the destruction of the CE-type antiferromagnetic order, causes sharp decrease of total thermopower. This means that the CE-type antiferromagnetic clusters with $\mathrm{CO}$ order are the main contributors to the thermopower of the whole sample.
\end{abstract}

\section{Introduction}

At the present time the practical application of thermoelectricity is predominantly confined by thermoelements for the temperature measurement. It is not used in other equipment like the thermoelectric generator, the refrigerator and the heaters for the heating because of low efficiency. As will be shown in our paper the usage of an inhomogeneous magnetic semiconductors can dramatically expand the boundaries of the use of thermoelectricity in applications.

In this paper, a wide temperature range investigated the thermoelectric power and influence it the magnetic field in the $\mathrm{Sm}_{0.55} \mathrm{Sr}_{0.45} \mathrm{MnO}_{3}$ manganite. Giant effects of the magnetoresistance and the volume magnetostriction were found in this compound previously [1]. Using neutron and electron diffraction and measuring the magnetic properties of the $\mathrm{Sm}_{0.5+\mathrm{x}} \mathrm{Sr}_{0.5-\mathrm{x}} \mathrm{MnO}_{3}(\mathrm{x}=0.05$, 0.10) system, authors of [2-5] found that it contains clusters of the following three types: ferromagnetic clusters, A-type antiferromagnetic clusters, and CE-type antiferromagnetic clusters. The ferromagnetic clusters concentrate charge carriers, but the A-type antiferromagnetic clusters haven't them at all. In the CEtype antiferromagnetic clusters takes place charge ordering (CO). In compound $\mathrm{Sm}_{0.55} \mathrm{Sr}_{0.45} \mathrm{MnO}_{3}$ ferromagnetic clusters have the Curie temperature $T_{C}=$ $126 \mathrm{~K}$, A-type antiferromagnetic clusters have the temperature Neel $T_{N A} \leq T_{C}$ and CE-type antiferromagnetic clusters have the temperature Neel $T_{N C E} \geq 180 \mathrm{~K}$.

\section{Experimental details}

Single-crystal $\mathrm{Sm}_{0.55} \mathrm{Sr}_{0.45} \mathrm{MnO}_{3}$ samples were grown by Balbashov using crucibleless zone melting and were cooled in an oxygen atmosphere. The phase compositions and the lattice parameters of the samples were controlled by Siemens D5000 X-ray diffractometer. The sample were single-phase perovskites with an orthorhombic structure (space group Pnma).

For the thermopower and magnetothermopower measurements a temperature gradient of $2 \mathrm{~K}$ across the sample was created using the electrical furnace on one end of the sample. There were used three previously graded copper-constantan thermocouples: to measure temperature difference between hot and cold ends of the sample and to measure temperature at the middle of the

\footnotetext{
a Corresponding author: koroleva@phys.msu.ru
} 
sample. Electrical measurements were carried out by analog-to-digital converter NI-9211.

The sample was placed on an evacuated inset which was droped in Dewar flask with liquid nitrogen. The sample temperature $T$ was regulated by electrical furnace rounded bifilarly on an inside surface of the evacuated inset. Dewar flask with the inset was disposed between poles of electromagnet. The magnetization of the sample was measured with SQUID magnetometer, and the paramagnetic susceptibility was measured by weighing with electromagnetic compensation.

Magnetothermopower and the magnetization $M$ measurements were carried out on two samples cut out from a single-crystal in the form of identical parallelepipeds. The longest edge was parallel to $c$-axis or $a b$-plane of an orthorhombic structure and parallel to vector of magnetic field $H$ during all measurements. $M(T)$ and $M(H)$ curves, as well as $\alpha(T)$ and $\alpha(H) ; \Delta \alpha / \alpha(T)$ and $\Delta \alpha / \alpha(H)$ were practically identical in two parallelepipeds indicating a small magnetic and crystallographic anisotropy in them.

\section{Results \& Discussion}

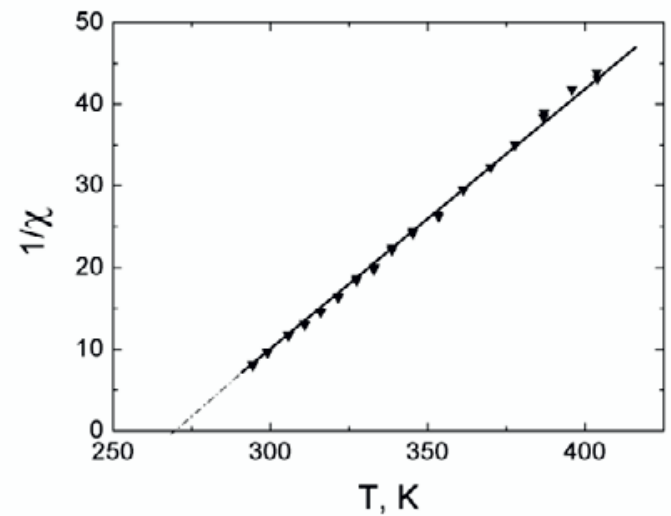

Figure 1. Temperature dependence of inverse paramagnetic susceptibility.

Figure 1 shows the temperature dependence of inverse paramagnetic susceptibility $1 / \chi$ of $\mathrm{Sm}_{0.55} \mathrm{Sr}_{0.45} \mathrm{MnO}_{3}$ sample. As can be seen from this figure, temperature dependence of $1 / \chi$ obeys the Curie-Weiss law with paramagnetic Curie temperature $\Theta=270 \mathrm{~K}$.

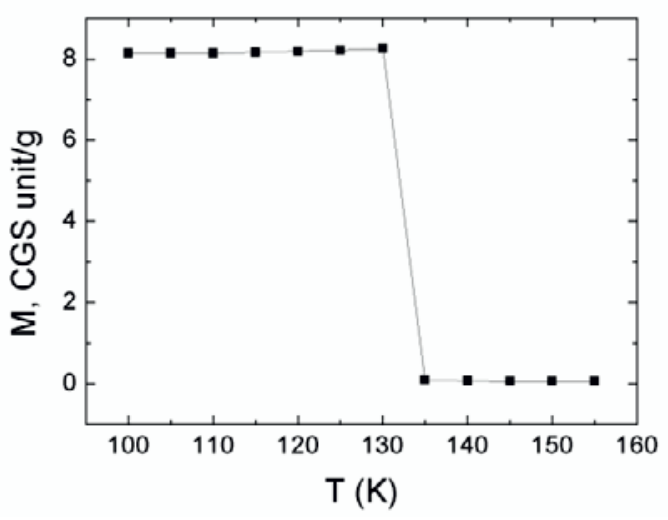

Figure 2. Temperature dependence of the magnetization in a magnetic field $\mathrm{H}=100$ Oe.

Figure 2 shows the temperature dependence of magnetization measured in a low field $H=100$ Oe. $M$ decreases sharply at a temperature of $134 \mathrm{~K}$; that is the Curie temperature of ferromagnetic clusters. It is close to the value $T_{C}=126 \mathrm{~K}$ determined in [2-5]. Thus, the value of $\Theta$ is significantly higher than $T_{C}$, what indicates to the presence of the CE-type antiferromagnetic clusters (whose sizes may change with temperature) in the temperature range $T_{C}<T<\Theta$. Annealing of sample in an oxygen atmosphere restores broken bonds $\mathrm{Mn}-\mathrm{V}-\mathrm{Mn}(\mathrm{V}$ is vacancy) and, thus, stabilizes the CE-type antiferromagnetic phase, increasing it's share in the sample.
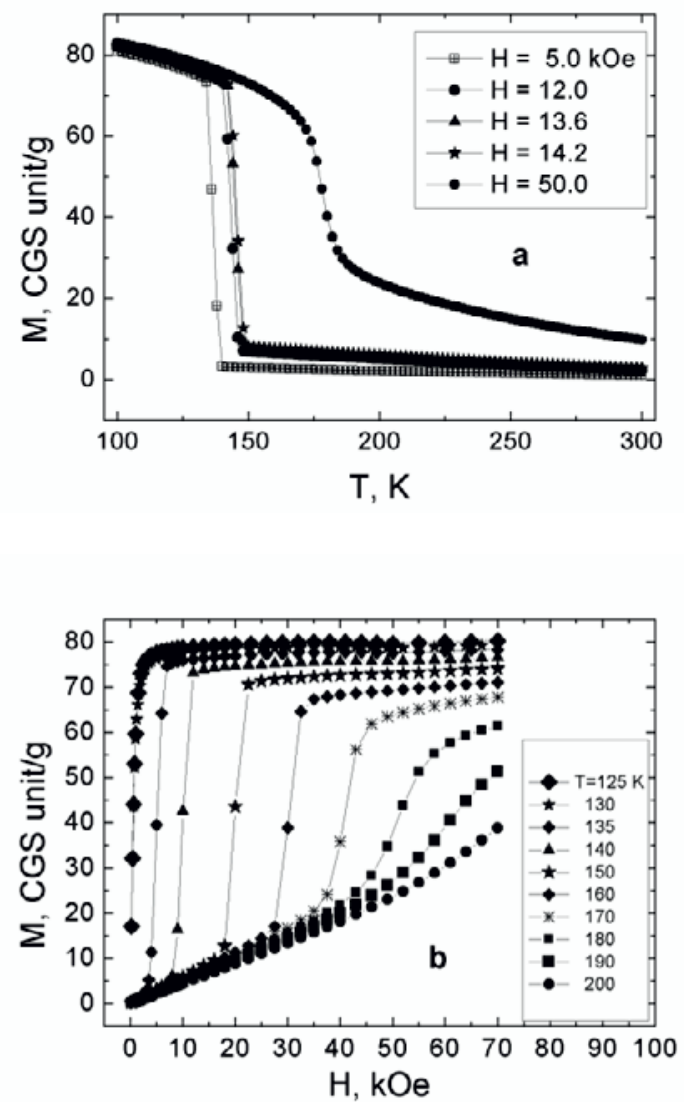


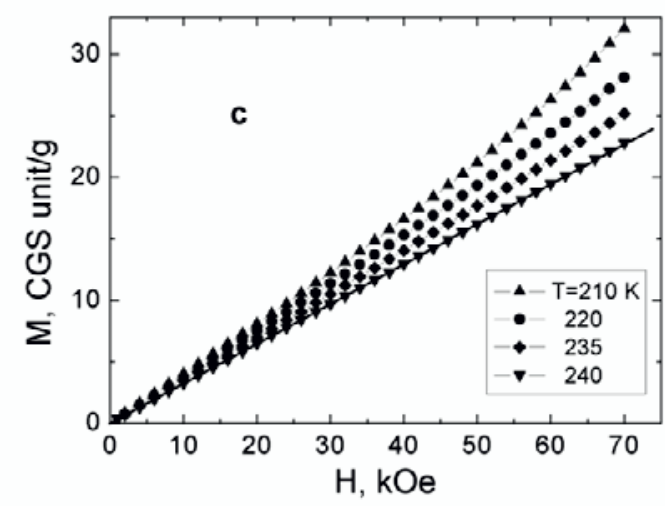

Figure 3. Magnetization vs. (a) temperature and (b), (c) magnetic field at various temperatures.

Figure 3 shows the temperature and field dependence of the magnetization in high magnetic fields (up to 70kOe). The transition from the spontaneous magnetization phase to the paramagnetic phase is strongly blurred (see Fig. 3a). As noted above, this is associated with the presence of the three types of magnetic clusters in the sample. The magnetization isotherms first linearely increase above the Curie point (Fig. 3b) due to the magnetization of A-type antiferromagnetic clusters and then increase sharply in the range $H_{C 1} \leq H \leq H_{C 2}$ due to the transition of CE-type antiferomagnetic clusters into the ferromagnetic state. The field dependence of the magnetization becomes linear at the temperature $T \sim 240 \mathrm{~K}$ (Fig. 3c), which can be considered as the temperature of breaking of the CEtype antiferromagnetic order $\left(T_{N C E}\right)$ and $\mathrm{CO}$ order. With the destruction of the last occurs a significant rearrangement of the crystal lattice, i.e., this is an analog of a first-order phase transition [6].

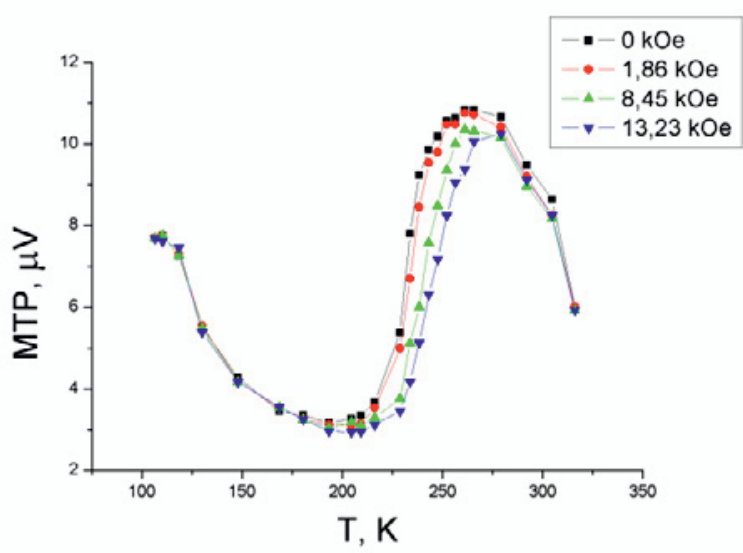

Figure 4. Temperature dependence of the thermopower in some magnetic fields.
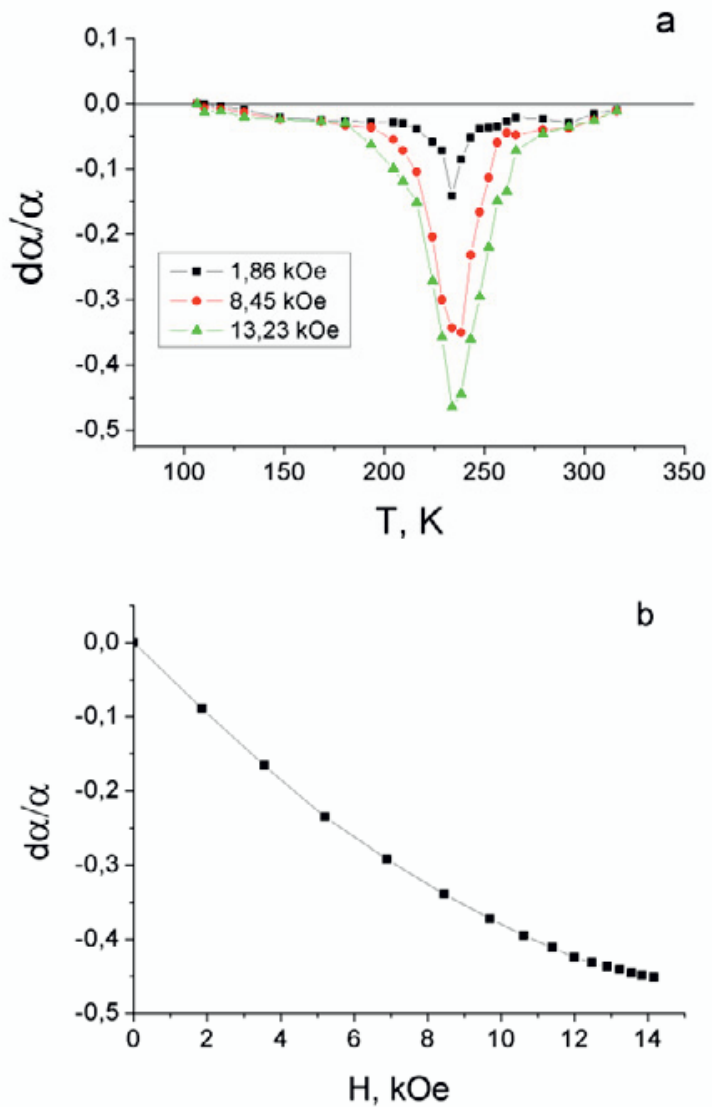

Figure 5. Magnetothermopower vs. (a) temperature and (b) magnetic field at temperature $240 \mathrm{~K}$.

The thermopower $\alpha$ and magnetothermopower temperature and magnetic field dependence $\Delta \alpha / \alpha=\left(\alpha_{H}-\right.$ $\left.\alpha_{H=0}\right) / \alpha$ were also studied in this work. Figure 4 shows the temperature dependence $\alpha(T)$ in some magnetic field and Figure $5 \mathrm{a}, \mathrm{b}$ shows the temperature dependence $\Delta \alpha / \alpha$ in some magnetic field (a) and dependence $\Delta \alpha / \alpha$ from $H$ at $T=240 \mathrm{~K}(\mathrm{~b})$. Graph on figure 4 has a broad maximum on the $\alpha(T)$ curves, which can be observed around $\Theta=$ $270 \mathrm{~K}$ including temperature $T_{N C E}=240 \mathrm{~K}$. Under the influence of the magnetic field this maximum decreases and shifts slightly to higher temperatures. Figure 5a shows that the sharp minimum in the $\{\Delta \alpha / \alpha\}(\mathrm{T})$ curves is located near to the $T_{N C E}$. At $T=T_{N C E}$ the $|\Delta \alpha / \alpha|$ reaches its maximum value nearly $50 \%$ in the magnetic field of $13,2 \mathrm{kOe}$. As Figure 5b shows the curve $\{\Delta \alpha / \alpha\}(H)$ at $T$ $=T_{N C E}$ does not saturates in the greatest measured field $13,2 \mathrm{kOe}$ and at $H>13,2 \mathrm{kOe}$ the $|\Delta \alpha / \alpha|$ may be higher. The sharp decrease of the value of $\alpha$ comes with the destruction of CE-type antiferromagnetic order and connected with its charge order. It also explains sharp decrease of magnetothermopower in strong magnetic fields.

From the above discussion it follows that thermopower in $\mathrm{Sm}_{0.55} \mathrm{Sr}_{0.45} \mathrm{MnO}_{3}$ compound at temperature, exceeded the Curie point of the ferromagnetic clusters, dues, mainly, the existence of CEtype antiferromagnetic clusters with the charge order displacing the oxygen ions. Altered crystalline lattice in 
these clusters provokes changes of the thermopower in them. Let the thermopower value in this clusters is $\alpha_{1}$ and outside is $\alpha_{2}$. Electrical current, flowed in sample at the $\alpha$-measurements, causes Peltier effect on boundaries of CE-type antiferromagnetic cluster (heating of one end and cooling of another end) and an overall temperature $\Delta \mathrm{T}$ arises on this cluster. Voltage created inside the cluster under influence of $\Delta T$ is equal to $\left(\alpha_{1}-\alpha_{2}\right) \Delta T$. Sharp decrease of the crystal thermopover $\alpha$ in $T_{N C E^{-}}$ region (Fig.5a) shows that the contribution of it from the antiferromagnetic CE-type clusters is big.

\section{Conclusions}

By this means magnetic inhomogeneous combined with strongly structural inhomogeneous influences on thermopower and magnetothermopower in $\mathrm{Sm}_{0.55} \mathrm{Sr}_{0.45} \mathrm{MnO}_{3}$. The value of thermopower may be regulated by magnetic field. Thus opened a new pathway for search of materials with big thermopower.

\section{Acknowledgment}

We thank A.M. Balbashov for the preparation and analysis of samples.

\section{References}

1. A.I. Abramovich, L.I. Koroleva, and A.V. Michurin, JETP 95 (5), 917 (2002)

2. V.V. Runov, D.Yu. Chernyshov, A.I. Kurbakov, et al, JETP 91(5) (2000)

3. C. Martin, A. Maignan, M. Hervieu, B. Raveau, phys. Rev. B 60, 12191 (1999)

4. V.V. Runov, G.P. Kopitsa, A.I. Okorokov et al, JETP Lett. 69 (4), 353 (1999)

5. V.V. Runov, H. Glattli Kopitsa G. et al, Physica, B276-278, 795 (221)

6. E. Dagotto, T. Hotta, A. Moreo, Phys. Rep., 344, $1(2001)$ 